\title{
Rate Maximization via PAPR Reduction in MIMO-OFDM Uplink: a User Cooperation Approach
}

\author{
Antti Arvola*, Antti Tölli*, and David Gesbert ${ }^{\dagger}$ \\ ${ }^{*}$ Centre for Wireless Communications, P.O. Box 4500, 90014 University of Oulu, Finland \\ E-mail: \{antti.arvola, antti.tolli\}@ee.oulu.fi \\ ${ }^{\dagger}$ EURECOM, Campus SophiaTech, 450 Route des Chappes, 06410 Biot Sophia Antipolis, France \\ E-mail: david.gesbert@eurecom.fr
}

\begin{abstract}
In this paper, we propose a novel user cooperation framework aimed at optimizing power efficiency in MIMOOFDM uplink, where cooperation is directed towards minimizing the transmit signal PAPR at all cooperating users. We consider different degrees of cooperation: 1) joint data transmission with PAPR reduction, 2) only joint data transmission and 3) the reference non-cooperative case based on a zero forcing receiver at the base station. In the cooperative case, we also account for the power required to exchange data between users. The main idea of our scheme is to exploit the unused space-frequency resources induced by the user cooperation to assign dummy symbols on the empty subcarriers and optimize these symbols to affect the outbound waveform. This is achieved as a result for a convex optimization problem which aims to simultaneously minimize the transmit PAPR and dummy symbol power allocation. We further introduce a rate maximization scheme, which maximizes the data power allocation for a given transmit peak power constraint by executing a one-dimensional search. The results show that our proposed PAPR minimizing bit and power allocation scheme can achieve significantly higher throughput at short and medium UEUE distances.
\end{abstract}

\section{INTRODUCTION}

MIMO-OFDM has become a well known and established technology in wireless communications, providing high data rates and high robustness against frequency selectivity. One of the major drawbacks of OFDM, however, is the high peakto-average power ratio (PAPR) induced by the inverse Fourier transform utilized by the scheme, especially when the number of subcarriers increases. To account for the high PAPR, the transceiver power amplifier (PA) operating point needs to have a backoff to accommodate the fluctuation in the signal power. Therefore the PA operates far in the linear region to avoid the distortion of the highest signal peaks (which cause adjacent channel power leakage), thus reducing the overall system power efficiency.

The PAPR reduction in OFDM is a well-known problem and an active research topic. Some popular methods of tackling the problem have been amplitude clipping as analyzed in

This research was supported by the Academy of Finland (Decision no. 279101 and 284590). D. Gesbert is supported by the ERC under the European Unions Horizon 2020 research and innovation program (Agreement no. 670896).
[1], selected mapping and partial transmit sequences [2]-[4] and constellation extension [5]. PAPR reduction in various optimization frameworks has also been studied in papers such as [6]-[8].

User cooperation in the form of cooperative transmission has been researched as a method to increase the robustness and reliability of wireless transmission in the form of user diversity, see for instance earlier work [9], [10]. Joint transmission of data can also increase the throughput of the uplink system by exploiting joint beamforming. In practice, user-side cooperation can be established using device to device (D2D) communications protocols, were the D2D link's efficiency typically depends on the inter-user distance. One important aspect of our work is that ability to account for inter-user distance in the power budget optimization.

The main contribution of this paper is the optimization framework aimed at reducing the transmit signal PAPR in a MIMO-OFDM uplink system. The key idea of our proposed framework is to utilize user cooperation induced unused spacefrequency resources (i.e., empty subcarriers) to transmit complex coefficients, dummy symbols, which can be ignored in the receiver. The idea of using unused resources for PAPR reduction is similar to [7], [8], however, instead of considering only peak power our scheme utilizes the strict PAPR expression of the transmit signal and in addition jointly minimizes the power allocated to the dummy symbols. The scheme described in this paper also focuses on (but is not limited to) conventional MIMO setting, whereas [7] considered the regime with very large antenna count.

Since the inverse discrete Fourier transform (IDFT) utilized by OFDM effectively scrambles all the symbols from different subcarriers to be present at every time instance in the time-domain transmit waveform, these dummy symbols can be optimized to reduce the PAPR of the final transmitted waveform. The optimization detailed in this paper also jointly minimizes the power required for the dummy symbols to be effective. The detailed method makes use of user cooperation in the form of joint transmission and joint beamforming to simultaneously maximize the uplink transmission rate and provide unused space-frequency resources for the optimization 
framework. This requires data exchange between users, which is modeled as a transmission over simple additive white Gaussian noise (AWGN) point-to-point channel. The transmit signal is formed using the well-known Hughes-Hartogs bit and power loading scheme [11], modifying the implementation of [12] for a MIMO setting. For transmit and receive beamformers, we consider a singular value decomposition (SVD) based approach in the cooperating case and zero forcing in the noncooperative case. Other methods of constructing the transmit signal or finding the transmit/receive beamformers are also valid, provided that the time-domain transmit waveform is known and there are space-frequency resources and power available for dummy symbol allocation. In fact, the method also works in the SISO case.

The PAPR reduction scheme with user cooperation is compared to cases where no user cooperation is present (only zero forcing receiver) and cooperation without PAPR optimization. These different methods are used to find the maximum rate achievable under a given peak power constraint (accounting for both the transmit power budget as well as the PAPR) and also evaluated with varying distances between the UEs (UE, user equipment). The latter evaluation provides insight into the tradeoff between the rates achieved via user cooperation and only zero forcing. The results show that by utilizing the PAPR reduction framework and user cooperation when user separation is small, the power allocated for data (i.e., transmitted rate) is significantly higher when compared to the case with no cooperation or no PAPR reduction. It can also be observed, that the non-cooperative zero forcing case starts to outperform the cooperating case when user separation increases enough, due to the high power requirement needed for data exchange and the increasing performance of the zero forcing receiver.

\section{System Model}

In this paper, we consider an uplink MIMO-OFDM system where a single base station (BS) with $M$ antennas serves $K$ single-antenna users on $N_{c}$ subcarriers. The users utilize transmit cooperation to enable transmit beamforming and joint data transmission. The cooperation also enables the use of the PAPR minimization framework detailed in Section III by providing additional mutually orthogonal space-frequency resources. For comparison, we also consider the case with no cooperation between users to highlight the benefits of our scheme. The detected signal on subcarrier $c$ and stream $s$ is given by

$\hat{x}_{c, s}=\mathbf{u}_{c, s}^{\mathrm{H}} \mathbf{H}_{c} \mathbf{v}_{c, s} \sqrt{p_{c, s}} x_{c, s}+\mathbf{u}_{c, s}^{\mathrm{H}} \mathbf{H}_{c} \sum_{\substack{i=1 \\ i \neq s}}^{K} \mathbf{v}_{c, i} \sqrt{p_{c, i}} x_{c, i}+\mathbf{u}_{c, s}^{\mathrm{H}} \mathbf{n}_{c}$

where the matrix $\mathbf{H}_{c}=\left[\begin{array}{ll}\mathbf{h}_{c, 1} & \mathbf{h}_{c, 2} \ldots \mathbf{h}_{c, K}\end{array}\right] \in \mathbb{C}^{M \times K}$ is the concatenation of different users' channel vectors on subcarrier $c$ and $\mathbf{u}_{c, s} \in \mathbb{C}^{M \times 1}$ and $\mathbf{v}_{c, s} \in \mathbb{C}^{K \times 1}$ denote the receive and transmit beamformers accessing stream $s$ on subcarrier $c$, respectively. The transmitted QAM-symbol is denoted by

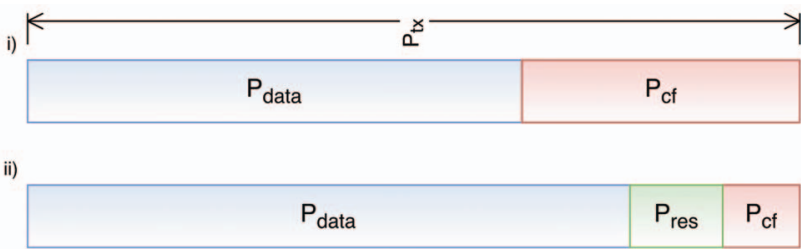

Fig. 1. Power allocation illustration. Graph i) illustrates the conventional power division and ii) has PAPR optimization applied.

$x_{c, s}$ with power normalized as $\mathbb{E}\left[\left|x_{c, s}\right|^{2}\right]=1, \forall c, s$, and the symbol power allocation is given separately by $p_{c, s}$. Finally, the $M$-length vector $\mathbf{n}_{c} \sim \mathcal{C N}\left(0, N_{0} \mathbf{I}\right)$ denotes the additive white Gaussian noise at the base station. Let us also denote the $N_{c} \times K$ allocation matrices for power and symbols as $\mathbb{P}$ and $\mathbb{X}$, respectively. The elements $(c, s)$ of these matrices contain the amount of power or the QAM-symbol allocated for subcarrier $c$ on spatial stream $s$.

Let us denote the logarithmic power allocation terms as follows: $P_{\mathrm{tx}}$ denotes the maximum allowed transmit peak power which includes the power allocated for data symbols $P_{\text {data }}$, dummy symbols $P_{\text {res }}$ and the transmit signal PAPR $P_{\text {cf }}$. The dummy symbol power allocation is considered as a factor of increase in $\mathrm{dB}$ on the data power allocation instead of a separate power term. Therefore, the relation between the logarithmic terms can be written as $P_{\mathrm{tx}}=P_{\text {data }}+P_{\text {res }}+P_{\text {cf }}$. Let us also denote the corresponding linear terms as $p_{\text {data }}, p_{\text {res }}$ and $p_{\mathrm{cf}}$, which will be further described in Section III. The power allocation scheme is illustrated in Figure 1.

The channel model utilized in this paper is the classical multipath model for uniform linear arrays [13]. The individual user channel vectors are expressed as

$$
\mathbf{h}_{c, k}=\frac{l_{d}}{\sqrt{L}} \sum_{l=1}^{L} \mathbf{a}\left(\alpha_{k, l}\right) e^{j \phi_{k, l}}, \forall c,
$$

where $l_{d}$ denotes the pathloss between the base station and the users, $L$ denotes the number of independent (and identically distributed, i.i.d) paths, $\phi_{k, l}$ is a random phase caused by the channel for path $l$, i.i.d. for different paths, and $\mathbf{a}(\alpha)$ is the array signature vector given by

$$
\mathbf{a}(\alpha)=\left[\begin{array}{c}
1 \\
e^{-j 2 \pi \frac{D}{\lambda} \cos (\alpha)} \\
\vdots \\
e^{-j 2 \pi \frac{(M-1) D}{\lambda} \cos (\alpha)}
\end{array}\right],
$$

where $D$ is the $\mathrm{BS}$ antenna spacing, $\lambda$ is the carrier wavelength and $\alpha$ is the angle of arrival (AoA). For simplicity, we assume that all the users are equidistant from the base station and the average pathloss is normalized as $l_{d}=1$.

In this paper, we utilize the Hughes-Hartogs bit and power allocation algorithm similar to [12] to construct the transmitted signal ${ }^{1}$. The algorithm provides an optimal bit and power

\footnotetext{
${ }^{1}$ The $\mathrm{HH}$ algorithm is not a prerequisite for the PAPR reduction framework. Other methods of constructing the transmitted time domain signal are also valid.
} 
allocation for a given bit error rate (BER) target and channel condition (thus requiring knowledge of the uplink channel) but requires zero interference. To eliminate interference between user streams, we consider singular value decomposition (SVD) based joint transmission when the users cooperate, and in a non-cooperative case the base station applies a zero forcing receiver to provide separation. When the users cooperate, we can decompose the channel matrix as $\mathbf{H}_{c}=\mathbf{U}_{c} \Lambda_{c} \mathbf{V}_{c}^{\mathrm{H}}$, and utilize the left and right eigenvectors as receive and transmit beamformers, respectively. In the case of no cooperation, a zero forcing receiver is applied at the base station, given by the pseudoinverse of the channel matrix as $\mathbf{U}_{c}=\mathbf{H}_{c}\left(\mathbf{H}_{c}^{\mathrm{H}} \mathbf{H}_{c}\right)^{-1}$.

The SVD based approach requires the exchange of data and channel state information (CSI) between the users. In this paper, we account only for the data exchange by assuming a simple AWGN channel between the users. We assume that this exchange happens by utilizing some out-of-band resources that do not interfere with the data transmission to the base station ${ }^{2}$. We further assume that the channel varies slowly enough so that the CSI exchange overhead is only a small fraction in comparison to the data exchange. This model can be used to calculate an additional power term required to obtain a certain joint transmission rate, based on the distance between the users relative to the distance from the base station. We assume that the users are at equal distance from the base station and have a separation between the users angles of arrival (AoA), denoted with $\theta$. Using this model, the distance between users becomes $\delta=2 d \sin \left(\frac{\theta}{2}\right)$, where $d$ is the distance from the base station. Now, assuming a pathloss exponent of $\gamma$, we can calculate the pathloss relation as a function of $\theta$ as

$$
\frac{l_{d}}{l_{\delta}}=\frac{1 / d^{\gamma}}{1 / \delta^{\gamma}}=\left(\frac{\delta}{d}\right)^{\gamma}=\left(2 \sin \left(\frac{\theta}{2}\right)\right)^{\gamma} .
$$

As mentioned above, the average pathloss between the users and base station is normalized to be $l_{d}=1$, which results in $l_{\delta}=\left(2 \sin \left(\frac{\theta}{2}\right)\right)^{-\gamma}$. The described model is illustrated in Figure 2.

The PAPR minimization problem requires knowledge of the time-domain transmitted signal from all transmit antennas. To simplify the final expression, let us denote the transmitted symbols and powers of all subcarriers on stream $s$, i.e., the column $s$ of matrices $\mathbb{X}$ and $\mathbb{P}$, with vectors $\mathbf{x}_{s} \in \mathbb{C}^{N_{c} \times 1}$ and $\mathbf{p}_{s} \in \mathbb{C}^{N_{c} \times 1}$, respectively. Furthermore, we can stack the transmit beamformers of all different subcarriers accessing stream $s$ to a single matrix as $\tilde{\mathbf{V}}_{s}=\left[\begin{array}{llll}\mathbf{v}_{1, s} & \mathbf{v}_{2, s} \ldots & \mathbf{v}_{N_{c}, s}\end{array}\right]^{\mathrm{T}} \in$ $\mathbb{C}^{N_{c} \times K}$. Using this notation, we can express the time-domain transmitted signals from all users with the $N_{c} \times K$ matrix

$$
\mathbf{S}^{\text {data }}=\mathbf{F}^{-1} \sum_{s=1}^{K} \mathbf{P}_{s}^{\frac{1}{2}} \mathbf{X}_{s} \tilde{\mathbf{V}}_{s}
$$

where $\mathbf{P}_{s}=\operatorname{diag}\left(\mathbf{p}_{s}\right), \mathbf{X}_{s}=\operatorname{diag}\left(\mathbf{x}_{s}\right)$ and $\mathbf{F}$ is the discrete Fourier transform (DFT) matrix.

\footnotetext{
${ }^{2}$ The case where the exchange happens on the same frequency band is an interesting case for future research.
}

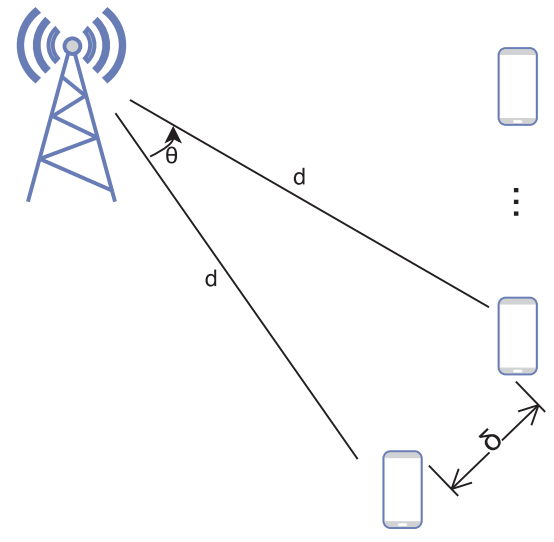

Fig. 2. Single-cell system illustration: multiple users at distance $d$ from the base station, with inter-user distance $\delta$ and angle $\theta$.

\section{OPTIMIZATION FRAMEWORK FOR PAPR REDUCTION}

In this section we describe our approach to minimizing the peak-to-average power ratio of the transmitted time domain signal in a MIMO-OFDM uplink system. ${ }^{3}$ The idea is to utilize the unused space-frequency resources to transmit dummy symbols which have an effect on the total transmit signal. Then, by optimizing these symbols, the transmit PAPR can be significantly reduced. The optimization framework also simultaneously aims to minimize the power allocated to these dummy symbols.

In this paper, we denote the logarithmic amount of power allocated to the dummy symbols as $P_{\text {res }}$ and PAPR as $P_{\mathrm{cf}}$ and the corresponding linear values with $p_{\text {res }}$ and $p_{\text {cf }}$, respectively. Now, we can formulate the optimization problem to minimize $P_{\text {res }}+P_{\text {cf }}$ in epigraph form as

$$
\begin{array}{cl}
\underset{\mathbf{M}, t, r}{\operatorname{minimize}} & 10 \log (t)+10 \log (r) \\
\text { subject to } & p_{\text {cf }} \leq t \\
& p_{\text {res }} \leq r \\
& {[\mathbf{M}]_{c, s}[\mathbb{P}]_{c, s}=0, \quad \forall c, s}
\end{array}
$$

where the matrix $\mathbf{M} \in \mathbb{C}^{N_{c} \times K}$ contains the dummy symbol allocation in a similar manner to $\mathbb{X}$. The complementary equality constraint ensures that dummy symbols are only allocated on elements of $\mathbf{M}$ where the corresponding element of $\mathbb{P}$ is zero, ensuring the utilization of empty subcarriers only. The problem in this form is non-convex and hence requires reformulation to be tractable.

The total time-domain transmit signal including the effect of $\mathbf{M}$ can be expressed in a manner similar to (5) as

$$
\mathbf{S}^{\text {total }}=\mathbf{S}^{\text {data }}+\mathbf{F}^{-1} \sum_{s=1}^{K} \operatorname{diag}\left(\mathbf{m}_{s}\right) \tilde{\mathbf{V}}_{s}
$$

where $\mathbf{m}_{s}$ denotes the column $s$ of matrix $\mathbf{M}$. Let us denote the column $k$ of $\mathbf{S}^{\text {total }}$ as $\mathbf{s}_{k}$ for notational simplicity. Now we

\footnotetext{
${ }^{3}$ The proposed scheme is not MIMO specific but works for OFDM in general.
} 
can express the PAPR of the time-domain transmit signal on antenna (or user) $k$ as

$$
p_{\mathrm{cf}, k}=\frac{\max _{i}\left(\left|s_{k, i}\right|^{2}\right)}{\operatorname{avg}\left(\mathbf{s}_{k}\right)}=\frac{N_{c}\left\|\mathbf{s}_{k}\right\|_{\infty}^{2}}{\left\|\mathbf{s}_{k}\right\|_{2}^{2}}
$$

and the power factor required to cover the dummy symbol power requirements as

$$
p_{\text {res }}=\frac{p_{\text {data }}+\|\mathbf{M}\|_{\mathrm{F}}^{2}}{p_{\text {data }}}=1+\frac{\|\mathbf{M}\|_{\mathrm{F}}^{2}}{p_{\text {data }}},
$$

where $p_{\text {data }}$ is the total power allocated for UE-BS data transmission and UE-UE data exchange. With these expressions the optimization problem becomes

$$
\begin{array}{cll}
\underset{\mathbf{M}, t, r}{\operatorname{minimize}} & \log (t)+\log (r) & \\
\text { subject to } & \frac{N_{c}\left\|\mathbf{s}_{k}\right\|_{\infty}^{2}}{\left\|\mathbf{s}_{k}\right\|_{2}^{2}} \leq t, & \forall k \\
& \|\mathbf{M}\|_{\mathrm{F}}^{2} \leq(r-1) p_{\text {data }} & \\
& {[\mathbf{M}]_{c, s}[\mathbb{P}]_{c, s}=0, \quad \forall c, s .}
\end{array}
$$

It is now apparent that the objective and the PAPR constraint are not valid convex expressions. For the PAPR constraint we can introduce the following slack variables: $w_{k}$ for the numerator and $q_{k}$ for the denominator, which operate as extra constraints $\left\|\mathbf{s}_{k}\right\|_{\infty} \leq w_{k}, \forall k$, and $q_{k} \leq\left\|\mathbf{s}_{k}\right\|_{2}^{2}, \forall k$. As a result, the PAPR constraint becomes convex (quadratic over linear), but the latter extra constraint is a difference of convex (DC) constraint and objective remains concave. Due to this reason, the problem can be solved using linearization by first order Taylor expansions and successive convex approximation (SCA), i.e., iterative optimization while updating a local point between every iteration, until convergence. The objective can be linearized around the local point $\{\tilde{t}, \tilde{r}\}$ as

$$
\log (t)+\log (r) \leq \log (\tilde{t})+\log \tilde{r}+\frac{1}{\tilde{t}}(t-\tilde{t})+\frac{1}{\tilde{r}}(r-\tilde{r})
$$

To linearize the RHS of the constraint $q_{k} \leq\left\|\mathbf{s}_{k}\right\|_{2}^{2}$, we first form a new vector $\mathbf{b}_{k}=\left[\operatorname{Re}\left(\mathbf{s}_{k}\right)^{\mathrm{T}} \operatorname{Im}\left(\mathbf{s}_{k}\right)^{\mathrm{T}}\right]^{\mathrm{T}}$, separating the real and imaginary parts of $\mathbf{s}_{k}$, and thus avoiding the non-linearities caused by the $l_{2}$-norm. Now, the RHS of the constraint $q_{k} \leq\left\|\mathbf{b}_{k}\right\|_{2}^{2}$ can be linearized around the local point $\left\{\tilde{\mathbf{b}}_{k}\right\}$ as

$$
\left\|\mathbf{b}_{k}\right\|_{2}^{2} \geq\left\|\tilde{\mathbf{b}}_{k}\right\|_{2}^{2}+2 \tilde{\mathbf{b}}_{k}^{\mathrm{T}}\left(\mathbf{b}_{k}-\tilde{\mathbf{b}}_{k}\right) .
$$

Utilizing the above formulation and omitting the constant terms, the final PAPR reduction problem can be expressed as

$$
\begin{array}{lll}
\underset{\mathbf{M}, \mathbf{b}_{k}, t, r, w_{k}, q_{k}}{\operatorname{minimize}} & \frac{r}{\tilde{r}}+\frac{t}{\tilde{t}} & \\
\text { subject to } & \frac{N_{c} w_{k}^{2}}{q_{k}} \leq t, & \forall k \\
& \left\|\mathbf{s}_{k}\right\|_{\infty} \leq w_{k}, & \forall k \\
& q_{k} \leq\left\|\tilde{\mathbf{b}}_{k}\right\|_{2}^{2}+2 \tilde{\mathbf{b}}_{k}^{\mathrm{T}}\left(\mathbf{b}_{k}-\tilde{\mathbf{b}}_{k}\right) & \forall k \\
& \|\mathbf{M}\|_{\mathrm{F}}^{2} \leq(r-1) p_{\text {data }} & \\
& {[\mathbf{M}]_{c, s}[\mathbb{P}]_{c, s}=0,} & \forall c, s
\end{array}
$$

Due to the non-convexity of the original problem, the global optimality of the result is not guaranteed. The PAPR reduction optimization is summarized in Algorithm 1.

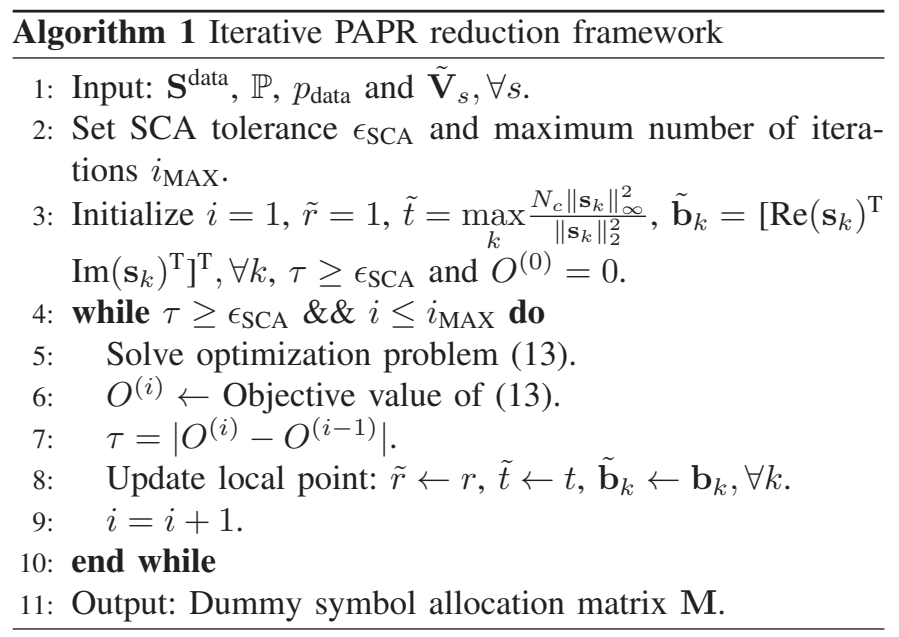

\section{Rate Maximization Under A Peak Power CONSTRAINT}

This section describes a rate maximization scheme that makes use of the PAPR reduction framework previously discussed. The transmit signal is constructed using the HughesHartogs bit and power loading algorithm. Our implementation follows that of [12] with a modification to operate in a multiantenna system while accounting for the power requirements of UE-UE data exchange. The algorithm iteratively allocates bits on subcarriers requiring the least amount of incremental power according to

$$
\Delta p_{c, s}=\left(2^{\Delta b}-1\right) 2^{b_{c, s}} \frac{5}{8} \ln \left(\frac{1}{5 \mathrm{BER}_{c, s}}\right) \mathrm{G}_{c, s}^{-1},
$$

where $\Delta b$ denotes the amount of bits increased every iteration, $b_{c, s}$ contains the current bit allocation of subcarrier $c$ on stream $s$ and $\mathrm{BER}_{c, s}$ is the subcarrier-wise target bit error rate ${ }^{4}$. The per-subcarrier channel gain term $\mathrm{G}_{c, s}$ depends on the level of user cooperation and is given by

$$
\mathrm{G}_{c, s}=\frac{\Gamma}{N_{0} B},
$$

where $B$ is the subcarrier bandwidth and $\Gamma=\sigma_{c, s}^{2}$ in the cooperative case and $\Gamma=\left\|\mathbf{u}_{c, s}\right\|_{2}^{-2}$ in the non-cooperative case. Here, $\sigma_{c, s}$ is the eigenvalue and $\mathbf{u}_{c, s}$ is the zero forcing receiver corresponding to stream $s$ on subcarrier $c$.

To account for the UE-UE data exchange, we need to add an additional power term to account for the increased traffic between users. The UE-UE exchange term can be calculated by dividing the total number of bits achieved by the UE-BS bit and power allocation as evenly as possible to all subcarriers of the UE-UE link, so that the number of exchanged bits on stream $s$, subcarrier $c$, follows $b_{c, s}^{\mathrm{UE}-\mathrm{UE}} \in\{0,2,4,6\}, \forall c, s$. Then the power requirement can be calculated subcarrier-wise with [14]

$$
p_{c, s}^{\mathrm{UE}-\mathrm{UE}}=\frac{5}{8}\left(2^{b_{c, s}^{\mathrm{UE}-\mathrm{UE}}}-1\right) \ln \left(\frac{1}{5 \mathrm{BER}_{c, s}}\right) \frac{\left|l_{\delta}\right|^{2}}{N_{0} B}
$$

\footnotetext{
${ }^{4}$ For simplicity, we consider a global BER target for all subcarriers
} 
The total power required for data exchange is the sum of these power terms over $c, s$, assuming for simplicity the same number of subcarriers as for the UE-BS link. In this paper, we consider the channel between the users, without loss of generality, to be flat fading and only dependent on the path loss between the users ${ }^{5}$. The described model calculates the minimum amount of power required to exchange all the data bits between users.

The extra power term is augmented into the bit and power allocation scheme, such that each increment in subcarrier bit allocation also covers the power required for data exchange. Then, bits are allocated iteratively until the data power constraint is met, resulting in an optimal allocation.

Our rate maximization scheme aims to allocate as much power as possible for $P_{\text {data }}$ by minimizing the sum $P_{\text {res }}+P_{\text {cf }}$ using the optimization framework described above. Therefore, as long as the minimized sum is smaller than the non-optimized PAPR, system throughput can be increased (see Figure 1). Our proposed scheme is a one-dimensional search for the best value of $P_{\text {data. }}$. It starts from the point $P_{\text {data }}=P_{\mathrm{tx}} / 2$ with the modified bit and power allocation, after which algorithm 1 is invoked to minimize $P_{\text {res }}+P_{\text {cf }}$. At this point, $P_{\text {data }}$ is also updated, since the bit and power loading allocates power in chunks. Now, the remaining usable power pool can be calculated as $P_{\text {rem }}=P_{\mathrm{tx}}-P_{\text {data }}-P_{\text {res }}-P_{\text {cf }}$ and allocated as extra power for data transmission. These steps are continued until the peak transmit power constraint is violated, after which we can backstep individual bit allocations and reoptimize until the peak power constraint is met. As PAPR is more sensitive to the number of subcarriers than the subcarrier power and symbol allocations, our scheme achieves the best fitting scenario in terms of $P_{\text {data }}$ rapidly. The rate maximization algorithm is summarized in Algorithm 2.

\section{NumericAl RESUlts}

In this section, the performance of our proposed optimization framework is investigated using the rate maximization search algorithm also outlined in this paper. We consider a system where a base station with $M=4$ antennas serves $K=2$ users simultaneously. The OFDM system has $N_{c}=64$ subcarriers and the transmitted symbols are selected randomly from the LTE uplink codebook, i.e., 4QAM, 16QAM and 64QAM. The BS-UE channel model has 40 distinct paths with 30 degree angular spread and the pathloss exponent is set to $\gamma=3$. For the optimization framework, SCA tolerance is set to $\epsilon_{\mathrm{SCA}}=10^{-3}$ with maximum iterations $i_{\mathrm{MAX}}=50$. For the bit and power loading algorithm, BER target is set to $10^{-3}$ while the bandwidth and noise power are normalized, i.e., $B=1$ and $N_{0}=1$.

The system throughput for different transmit peak power constraints is plotted in Figure 3. The rate is averaged over 5000 symbol realizations (100 channel realizations with 50 symbol realizations each) and plotted for both cooperative and

${ }^{5} \mathrm{~A}$ fading case can also be considered, where the UE-UE link also utilizes bit and power loading to obtain subcarrier-wise bit allocation. $\overline{\text { Algorithm } 2 \text { Rate maximization search algorithm with bit and }}$ power allocation for a given transmit peak power $P_{\mathrm{tx}}$ with user cooperation

1: Input: $P_{\mathrm{tx}}$.

2: Set $P_{\text {data }}=P_{\mathrm{tx}} / 2, P_{\mathrm{cf}}=0, P_{\text {res }}=0$.

3: while $P_{\text {data }}+P_{\mathrm{cf}}+P_{\text {res }}<P_{\mathrm{tx}}$ do

4: Calculate optimal bit and power allocation while accounting for the power requirement of data exchange.

5: $\quad$ Update $P_{\text {data }}$, as bit and power loading allocates power in chunks.

6: $\quad$ Optimize PAPR with Algorithm 1.

7: $\quad$ Calculate PAPR $\left(P_{\mathrm{cf}}\right)$ and power increment allocated to dummy symbols $\left(P_{\text {res }}\right)$.

8: $\quad$ Remaining power: $P_{\text {rem }}=P_{\mathrm{tx}}-P_{\text {data }}-P_{\mathrm{cf}}-P_{\mathrm{res}}$.

9: $\quad$ Adjust data power allocation: $P_{\text {data }}=P_{\text {data }}+P_{\text {rem }}$.

10: end while

11: Backstep individual bit allocations and re-optimize until $P_{\text {data }}+P_{\text {cf }}+P_{\text {res }}<P_{\text {tx }}$.

12: Output: Optimized bit and power allocation for given peak transmit power $P_{\mathrm{tx}}$.

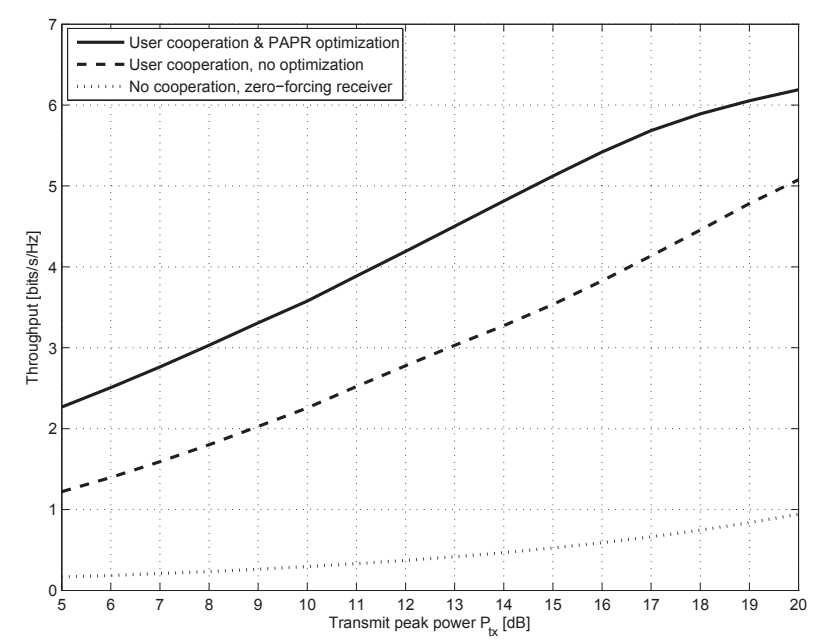

Fig. 3. System throughput as a function of transmit peak power.

noncooperative case. The angle between users is set to $\theta=$ $10^{\circ}$. From the picture it is obvious that the PAPR reduction scheme can provide significant throughput gains for constant $P_{\mathrm{tx}}$. This is due to the suppressed transmit PAPR, which allows for more power allocated for data transmission. It can also be seen that the throughput starts to saturate on higher $P_{\mathrm{tx}}$ when PAPR reduction is used, while zero forcing performs badly due to low angular separation (high correlation) of users.

Focusing on the points $P_{\mathrm{tx}}=\{10,20\} \mathrm{dB}$, the complementary cumulative distribution function $(\mathrm{CCDF})$ of the transmit PAPR is plotted in Figure 4. The CCDF is simulated over 10000 symbol realizations (100 channels, 100 symbols per channel). From the graph it can be seen that our proposed optimization framework can provide significant transmit PAPR suppression when compared to the OFDM baseline. For example, even at transmit peak power of $P_{\mathrm{tx}}=20 \mathrm{~dB}$ the PAPR 


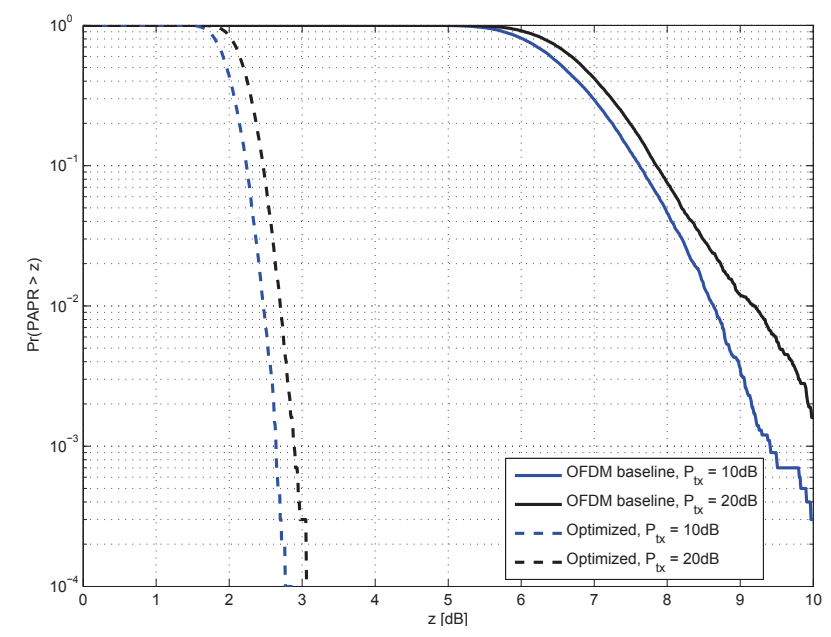

Fig. 4. Complementary cumulative distribution function of the transmit signal PAPR with and without PAPR optimization for transmit peak powers $P_{\mathrm{tx}}=$ $\{10,20\} \mathrm{dB}$. The optimized PAPR includes the power allocated for dummy symbols, i.e., $P_{\mathrm{cf}}+P_{\text {res }}$.

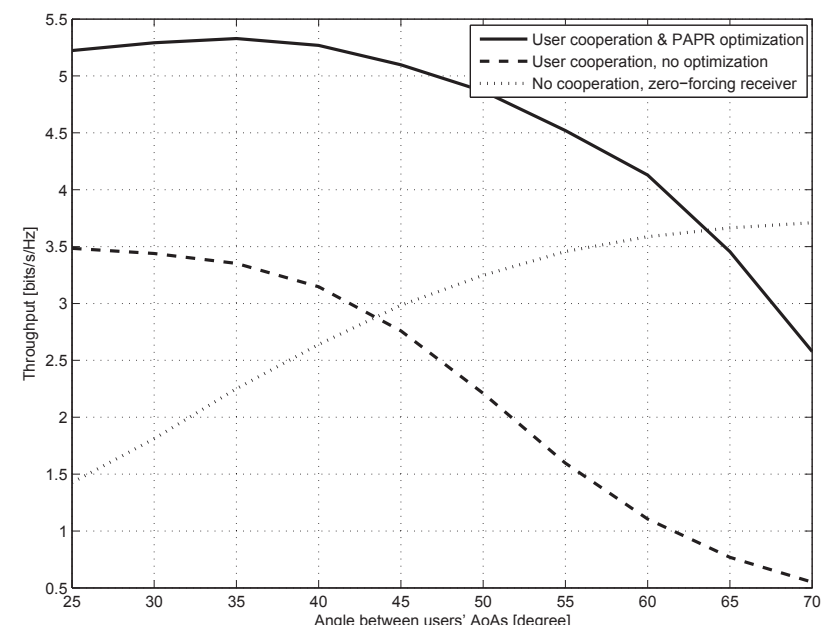

Fig. 5. The throughput performance as a function of the angle $\theta$ between users (i.e., the distance between users).

barely reaches $3 \mathrm{~dB}$ while unoptimized it can be as high as 10 $\mathrm{dB}$. We can also note that the transmit peak power constraint has a small effect on the PAPR, as higher powers fill more subcarriers with symbols of higher amplitude.

To gain insight into the tradeoff between cooperative and noncooperative transmit schemes in terms of the distance between users, we choose $P_{\mathrm{tx}}=15 \mathrm{~dB}$ as transmit peak power and simulate the throughput performance as a function of the angle $\theta$. We consider the range $\theta \in[25,70]^{\circ}$, where each point is averaged over 1000 symbol realizations (40 channels with 25 symbols each). The results are presented in Figure 5. From the figure we can see the point where user cooperation is no longer beneficial. With greater distances the angular resolution of the $\mathrm{BS}$ antenna array provides better gains for the zero forcing receiver, while in the cooperative case the UEUE data exchange requires ever more power. Using the PAPR reduction scheme the crossing point is at greater distance, due to the higher power allocated for data. When the users are co-located, the user cooperation and PAPR reduction provide significant improvements in throughput. The slight increase in throughput from $\theta=25^{\circ}$ to $\theta=35^{\circ}$ while utilizing the PAPR minimization scheme is due to the combined effects of higher data power allocation and lower user correlation.

\section{CONCLUSIONS}

In this paper, we proposed an optimization framework to minimize the transmit signal peak-to-average power ratio by utilizing the unused space-frequency resources of the OFDM system. The PAPR reduction scheme is enabled by user cooperation in the form of joint transmission, which requires data exchange between the users. Our approach utilized the well-known Hughes-Hartogs bit and power allocation scheme for transmit signal construction. The proposed optimization framework was co-opted in a rate maximization search algorithm also proposed in this paper. The simulation results showed significant gains in terms of throughput if the PAPR reduction framework is exploited.

\section{REFERENCES}

[1] F. Peng and W. Ryan, "On the capacity of clipped OFDM channels," in Information Theory, 2006 IEEE International Symposium on, July 2006, pp. 1866-1870.

[2] R. Bauml, R. Fischer, and J. Huber, "Reducing the peak-to-average power ratio of multicarrier modulation by selected mapping," Electronics Letters, vol. 32, no. 22, pp. 2056-2057, Oct 1996.

[3] R. F. H. Fischer and M. Hoch, "Directed selected mapping for peak-toaverage power ratio reduction in MIMO OFDM," Electronics Letters, vol. 42, no. 22, pp. 1289-1290, Oct 2006.

[4] S. Muller and J. Huber, "OFDM with reduced peak-to-average power ratio by optimum combination of partial transmit sequences," Electronics Letters, vol. 33, no. 5, pp. 368-369, Feb 1997.

[5] B. S. Krongold and D. L. Jones, "PAR reduction in OFDM via active constellation extension," IEEE Transactions on Broadcasting, vol. 49 no. 3, pp. 258-268, Sept 2003.

[6] V. Tervo, A. Tölli, and T. Matsumoto, "PAPR constrained power allocation for multicarrier transmission in multiuser SIMO communications," IEEE Transactions on Wireless Communications, vol. 15, no. 4, pp. 2458-2473, April 2016.

[7] C. Studer and E. G. Larsson, "PAR-aware large-scale multi-user MIMOOFDM downlink," IEEE Journal on Selected Areas in Communications, vol. 31, no. 2, pp. 303-313, February 2013.

[8] H. Zhang and D. L. Goeckel, "Peak power reduction in closed-loop mimo-ofdm systems via mode reservation," IEEE Communications Letters, vol. 11, no. 7, pp. 583-585, July 2007.

[9] A. Sendonaris, E. Erkip, and B. Aazhang, "User cooperation diversity. part i. system description," IEEE Transactions on Communications, vol. 51, no. 11, pp. 1927-1938, Nov 2003.

[10] — , "Increasing uplink capacity via user cooperation diversity," in Information Theory, 1998. Proceedings. 1998 IEEE International Symposium on, Aug 1998, pp. 156-.

[11] D. Hughes-Hartogs, "Ensemble modem structure for imperfect transmission media," May 23 1989, uS Patent 4,833,706.

[12] Q. Sun, L. Li, A. Tölli, M. Juntti, and J. Mao, "Optimal energy efficient bit and power loading for multicarrier systems," Communications Letters, IEEE, vol. 18, no. 7, pp. 1194-1197, July 2014.

[13] A. F. Molisch, Wireless communications. John Wiley \& Sons, 2007.

[14] S. T. Chung and A. Goldsmith, "Degrees of freedom in adaptive modulation: a unified view," Communications, IEEE Transactions on, vol. 49, no. 9, pp. 1561-1571, Sep 2001. 